\title{
Experiencia perceptual y sustento epistémico ${ }^{1}$
}

\section{(Perceptual experience and epistemic support)}

\author{
José Luís Falguera y Santiago Peleteiro
}

Recibido: 11 de mayo de 2012

Aceptado: 26 de junio de 2013

\section{Resumen}

En este trabajo analizamos el problema de cómo nuestras experiencias perceptuales sustentan epistémicamente nuestras creencias. Partimos de algunas consideraciones de Fodor al respecto, aunque revisadas en ciertos aspectos. Especialmente nos ocupamos de su reciente defensa de la existencia de representaciones no-conceptuales en el procesamiento de la información perceptual. Nuestra pretensión es reivindicar, frente a los argumentos en contra esgrimidos por algunos representantes del llamado 'conceptualismo perceptual', un papel normativo para las experiencias perceptuales concebidas como representaciones con contenido no-conceptual.

Palabras clave: experiencia perceptual, representación icónica, contenido noconceptual, justificación, legitimación epistémica, sustento epistémico.

\begin{abstract}
In this paper we analyze the problem of how our perceptual experiences give epistemic support to our beliefs. We begin with some considerations formulated by Fodor, but revise them in some respects. We consider, in particular, his recent proposal for the existence of non-conceptual representations in perceptual information processing. Our aim is to vindicate, against the arguments raised by some represen-

1 Este trabajo participa de los proyectos de investigación FFI2013-41415-P, financiado por el Ministerio de Economía y Competitividad (de España), y PTDC/FIL-FIL/109882/2009, financiado por la Fundação para a Ciência e a Tecnologia (Ministério da Ciência, Tecnologia e Ensino Superior de Portugal). Agradecemos las sugerencias de dos comentaristas anónimos que nos han permitido introducir mejoras sobre una versión previa de este trabajo. En todo caso es de nuestra exclusiva responsabilidad toda deficiencia que aún perdure.
\end{abstract}


tatives of so-called 'perceptual conceptualism', a normative role for perceptual experiences insofar as they are conceived as representations with non-conceptual content.

Keywords: perceptual experience, iconic representation, non-conceptual content, justification, epistemic entitlement, epistemic support.

\section{Introducción}

Uno de los problemas tradicionales de la epistemología contemporánea radica en establecer qué papel juegan nuestras experiencias perceptuales (en adelante, EPs) en la formación y justificación de creencias, y en particular en la justificación de nuestras creencias de experiencia más básicas o creencias de experiencia perceptual (en adelante, CEPs). ${ }^{2}$ El cuestionamiento por parte de Sellars (1963) de que lo dado pueda servir para justificar creencias propició planteamientos sobre este asunto en los que se ha relegado a las EPs al cumplimiento de un rol meramente causal. No obstante, la posibilidad de que las EPs ejerzan algún papel epistémico normati$v o$ respecto a ciertas creencias se ha convertido en las últimas décadas en un punto relevante de la controversia entre conceptualistas y no-conceptualistas acerca del contenido de la percepción. ${ }^{3}$ Los primeros creen poder reasignarle ese papel alejando a las EPs de su carácter de lo dado al concebirlas como conceptuales, atribuyendo al mismo tiempo a sus rivales en el debate, como Peacocke (1992; 2001), una cierta rehabilitación normativa de lo dado que consideran improcedente. Desde la perspectiva de los conceptualistas la solución al problema del papel normativo de las EPs pasa por aceptar que ya en ellas se ponen en juego las mismas capacidades conceptuales que se requieren para fijar creencias sobre aquellos estados de cosas que dichas experiencias ponen de manifiesto. Esta propuesta conlleva asumir que el contenido de una EP debe ser proposicional o estar conceptualmente estructurado. Veremos que autores referentes en ésta línea, como McDowell (1994a) o Brewer $(1999 ; 2005)$, consideran que ese carácter conceptual es la condición necesaria para que las EPs puedan justificar creencias y ejercer así un papel epistémico normativo. ${ }^{4}$ La primera sección de este trabajo estará dedicada a exponer brevemente los

\footnotetext{
2 Por 'creencia perceptual' entendemos aquella creencia que un sujeto tiene acerca de una escena (sus entidades, propiedades y relaciones) en tanto que ese sujeto tiene (o ha tenido) alguna EP de dicha escena.

3 Para una visión panorámica de este debate puede consultarse el trabajo de Toribio (2009). Por otra parte, Bermúdez y Cahen (2003/2010) proporcionan una buena introducción a la teoría del contenido no-conceptual.

4 Es necesario matizar que McDowell (2009), aunque sigue manteniendo una postura conceptualista, ha abandonado dos de las tesis fundamentales que sostenía en su propuesta de 1994. Así, por un lado
} 
argumentos desarrollados por estos autores. La cerraremos afirmando que dichos argumentos sólo resultan concluyentes para quien de entrada asume una noción general de 'contenido de un estado mental' que se nos antoja restringida, en virtud de la cual todo contenido atribuible a un sujeto está determinado por los conceptos que éste posee..$^{5}$

Frente a esta posición conceptualista, nosotros entendemos que postular el contenido no-conceptual de las EPs es compatible con atribuirles un rol normativo. Nuestra intención es formular una propuesta sobre cómo las EPs con contenido noconceptual apoyan CEPs. Para ello partiremos, en una segunda sección, de algunos planteamientos de Fodor con respecto a las EPs. Si bien este autor mantiene su tradicional posición de que las EPs son conceptuales, recientemente (Fodor, 2007) ha planteado que podemos encontrar un apoyo epistémico para nuestras CEPs en un tipo de representaciones no-conceptuales que tienen lugar en fases tempranas del proceso de percepción. Tales representaciones se darían por lo tanto a un nivel subpersonal y Fodor las considera como lo dado en dicho proceso de percepción. Analizaremos y asumiremos estos planteamientos de Fodor, si bien distanciándonos de su idea de atribuir un carácter subpersonal a las representaciones no-conceptuales que ha postulado. Será en una tercera y última sección en donde partiendo de este y otros planteamientos de Fodor expondremos nuestra propia propuesta basada en lo que denominaremos "sustento epistémico"; una relación epistémica entre las EPs y las CEPs, que no ha de confundirse con la postulada clásica relación inferencial de justificación. Con la noción de 'sustento epistémico' optamos por una manera diferente de entender la relación normativa entre EPs y CEPs. La relación de sustento epistémico es aparentemente similar a una considerada en los planteamientos de Burge (2003), Peacocke (2004) o Pryor (2005). En estos trabajos se habla de la relación normativa entre EPs y CEPs en términos de "legitimación" o de "justificación inmediata". No obstante, veremos que nuestra noción y las de ellos no coinciden plenamente, e indicaremos las diferencias.

rechaza la idea de que el contenido de las EPs posea estructura proposicional, abogando ahora por lo que denomina "contenido intuicional". Dicho contenido en todo caso aun es conceptual para McDowell porque «cada aspecto del contenido de una intuición está presente de tal forma que ya es adecuado para ser el contenido asociado a una capacidad discursiva, si no está - al menos no aún efectivamente así asociada» (McDowell, 2009, p. 264). Por otro lado, también rechaza la idea de una relación de justificación inferencial entre EPs y CEPs, hasta el punto de negar que con anterioridad hubiese defendido dicha idea, en contra de la interpretación que suele ser habitual (McDowell, 2009, p. 270). De todos modos, pese a este cambio de postura, aquí centraremos nuestra atención en sus planteamientos de 1994.

5 Aunque es cierto que, salvo alguna excepción, existe un notable acuerdo entre conceptualistas y noconceptualistas en abordar la cuestión del contenido de los estados de creencia en términos de proposiciones (y los conceptos que las constituyen), para los conceptualistas también los contenidos de las EPs han de ser de esa índole al adoptar la mencionada noción general de contenido. 


\section{Conceptualismo perceptual y justificación de creencias}

1. La motivación fundamental de la propuesta conceptualista es, como indica McDowell (1994a, Introducción), tratar de salvaguardar el carácter normativo de la relación entre mente y mundo, entendiendo esto como la necesidad de que el pensamiento que establece un juicio o fija una creencia acerca del mundo responda ante éste o sea evaluable en función de cómo son las cosas. El éxito de esa tarea depende en la opinión de este autor del modo en que se logre entender la relación entre el pensamiento empírico y la experiencia, en especial entre CEPs y EPs. Esa relación debería concebirse como aquella en la cual el pensamiento empírico se ve sometido por parte de la experiencia a una constricción evaluativa de carácter racional. Dicho en términos quineanos, la experiencia perceptual debería poder cumplir un papel de tribunal. La dificultad de asignarle un papel como ese radica, según McDowell, en el hecho de que continuemos manejando una concepción de 'experiencia' heredada del empirismo tradicional y entendida como mera presencia, conjunto de impresiones o simples impactos sobre nuestro sistema sensorial. Su diagnóstico es que al persistir en esa concepción nos vemos obligados a oscilar continuamente entre dos extremos incompatibles, a su modo de ver, con una interpretación normativa cabal del papel de la experiencia perceptual: por un lado, hacia un coherentismo puro que termina rechazando cualquier papel de la misma que no sea el meramente causal; por otro, hacia propuestas que si bien le atribuyen un papel de justificación, entiende que no resultan viables por su incapacidad para caracterizar la relación entre experiencia y creencia como una relación racional. En ambos casos, opina, se torna misterioso el contacto entre pensamiento y mundo empíricos.

Como paradigma del primero de estos extremos McDowell señala a Davidson y su tesis-eslogan conforme a la cual "nada puede contar como razón para sostener una creencia sino otra creencia" (Davidson, 1983, p. 126). Según esto, lo único que pueden hacer nuestras EPs es causar creencias, nunca justificarlas:

The relation between a sensation and a belief cannot be logical, since sensations are not beliefs or other propositional attitudes. What then is the relation? The answer is, I think, obvious: the relation is causal. Sensations cause some beliefs and in this sense are the basis or ground of those beliefs. But a causal explanation of a belief does not show how or why the belief is justified. (Davidson, 1983, p. 127).

Tras esta tesis resuenan las consideraciones de Sellars (1963, §§ 32-38) en contra del enfoque fundamentista de la justificación de creencias y de su pretensión de encontrar el sustento último de nuestro conocimiento en algo concebido como lo dado o que no involucra habilidades conceptuales. Sellars quiso demostrar que tal pretensión no es más que una versión de lo que conocemos como 'mito de lo dado'. Davidson comparte ese rechazo a considerar lo dado como algo válido para justifi- 
car, hasta el punto de que McDowell (1994a, pp. xv-xvi) presenta a ambos autores como intercambiables. ${ }^{6}$ En efecto, al concebir las EPs como algo dado -es decir, como mero conjunto de impresiones o impactos sobre nuestra sensibilidad ambos autores entienden que debería ser excluida de lo que el propio Sellars (1963, p. 169) denominó 'espacio lógico de las razones' o ámbito donde se llevan a cabo justificaciones. Concebidas como impresiones, las EPs no son para ellos más que meras transacciones dentro de un ámbito completamente distinto: el ámbito de lo natural o 'espacio lógico de las leyes naturales', donde lo que se establecen son relaciones causales. Esa exclusión del espacio de las razones conllevaría en última instancia una renuncia al empirismo entendido como la defensa de la idea de que la experiencia constituye el tribunal al que se someten nuestras creencias empíricas (McDowell, 1994a, p. xviii).

Como ejemplo del segundo de los extremos citados McDowell hace referencia a las propuestas de Evans (1982, pp. 227-228) y Peacocke (1992, p. 80; 2001, pp. 254 ys.), en las que se considera que nuestras EPs poseen un contenido no-conceptual y que a pesar de ello pueden ser entendidas como razones en base a las que justificar CEPs. ${ }^{7}$ Con estas últimas propuestas se amplía el espacio de las razones más allá del espacio de lo conceptual. Sin embargo, esto a ojos de McDowell (1994a, pp. 7-8) constituye una recaída en el mito de lo dado. ${ }^{8} \mathrm{~A}$ su entender, un contenido noconceptual pertenece más bien al espacio lógico de lo natural; espacio que Sellars (1963, p.169) presentaba como un ámbito de cuyos elementos se pueden ofrecer descripciones empiricas, pero no extraer justificaciones. Bajo esta óptica, si se deja el contenido perceptual fuera de la esfera de lo conceptual, el no-conceptualista sólo podrá ofrecer descripciones de lo que se requiere para creer (del supuesto contenido no-conceptual), pero no podrá dar cuenta de lo que realmente desea explicar, a saber: que dicho contenido perceptual "pueda constituirse inteligiblemente como las razones de un sujeto para llegar a creer algo" (McDowell, 1994a, p. 163). McDowell (1994a, p. 166) no ve cómo las EPs, entendidas como no-conceptuales, podrían ser valoradas por el propio sujeto como razones de sus creencias. Para él dicha valoración implica que el sujeto sea capaz de una comprensión exhaustiva que abarque tanto el contenido de sus EPs como el de las correspondientes CEPs en términos de los conceptos que posee. Atribuir un contenido no-conceptual a las primeras impide, en su opinión, que tal comprensión exhaustiva se produzca. McDowell

\footnotetext{
${ }^{6}$ Cabe matizar que no se puede atribuir a Sellars un coherentismo tan contundente como el que Davidson adopta (véase Sellars, 1963, p. 170).

7 Como se verá más adelante, en Peacocke (2004) se constata un cambio de posición respecto de sus trabajos de 1992 y 2001.

8 Debemos aclarar que en el caso de Evans la acusación de recaída en el mito de lo dado es matizada por el propio McDowell debido a la peculiar concepción que aquél tiene de 'experiencia'. Para Evans las EPs poseen contenido no-conceptual, pero sólo pueden considerarse experiencias por estar a disposición de un sujeto con capacidades conceptuales (Evans, 1982, pp. 157-158; McDowell, 1994a, p. 49).
} 
entiende que ese contenido perceptual, en tanto que no estaría conceptualmente estructurado, resultaría opaco en el sentido de que el sujeto no sería capaz de apreciar adecuadamente el modo en el que el mundo se le presenta en dicha experiencia. ${ }^{9}$ McDowell concuerda aquí con Davidson en defender que «el hecho de que a uno se le muestren las cosas de tal o cual modo es algo que ya se encuentra, como tal, dentro del espacio de los conceptos» (1994a, p. 139). Siendo así, las EPs noconceptuales no constituirían razones propiamente dichas y su relación con las CEPs no podría ser caracterizada como una relación racional.

En definitiva, ninguno de los dos extremos anteriores -representados respectivamente por el coherentismo de Davidson y las versiones no-conceptualistas de Evans (1982) y Peacocke (1992; 2001)- permiten, según McDowell, entender las EPs como tribunal. Y dado que en ambos casos el problema reside para él en la adopción de una concepción tradicional de experiencia que la excluye del ámbito lógico de las razones, propone como solución reintroducirla en ese ámbito simplemente reconociendo que su contenido es conceptual, es decir, admitiendo que ya a nivel de nuestras EPs se actualizan los mismos conceptos que configuran el contenido de nuestras CEPs. ${ }^{10} \mathrm{McD}$ owell considera que aceptar esto facilita presentar la experiencia perceptual como una genuina apertura al mundo, haciendo perfectamente inteligible la manera en que cualquier sujeto evalúa sus creencias en base a ella. Su estrategia consiste en que, aún asumiendo que las EPs no son creencias, si les atribuimos un contenido del mismo tipo podrán ejercer las mismas funciones de justificación que Davidson reservaba exclusivamente a las creencias. De hecho, McDowell propone reformular la tesis de Davidson diciendo que "nada puede contar como una razón para sostener una creencia salvo alguna otra cosa que también se encuentre en el espacio de los conceptos." (McDowell, 1994a, pp. 140 y 143), De ese modo, opina, dicha tesis dejaría de constituir un obstáculo para una concepción normativa cabal del papel de las EPs. La ventaja de la solución conceptualista sería pues que, de ser correcta, nos permitiría entender la relación entre EPs y CEPs como indiscutiblemente racional.

\footnotetext{
${ }_{9}$ McDowell asume sin reservas la segunda parte del aforismo kantiano según el cual los conceptos sin intuiciones están vacíos mientras que las intuiciones sin conceptos son ciegas: "To say that an experience is not blind is to say that it is intelligible to its subject as purporting to be awareness of a feature of objective reality: as a seeming glimpse of the world." (McDowell, 1994a, p. 54).

10 Esta maniobra implica difuminar la línea divisoria entre el espacio lógico de las leyes naturales y el espacio lógico de las razones., lo que podría dar lugar a acusaciones de idealismo. Con el fin de evitarlas McDowell (1994a, pp. xviii y ss.) echa mano de la noción de 'segunda naturaleza', aquella que "los seres humanos adquieren al ser adiestrados en las capacidades conceptuales" (p. xx). Su idea es que toda vez que entendamos que por su naturaleza la especie humana se relaciona con su entorno fundamentalmente a través del uso de capacidades conceptuales no debería resultar difícil concebir nuestras EPs como algo que pertenece al ámbito lógico de las razones sin dejar de pertenecer al ámbito de lo natural.
} 
2. En Brewer (1999 y 2005) nos encontramos con unos planteamientos similares, desarrollados al hilo de un argumento que simplificado reza como sigue:

(1) Las experiencias sensoriales proporcionan razones para las creencias empíricas.

(2) Las experiencias sensoriales proporcionan razones para las creencias empíricas sólo si tienen contenido conceptual.

$\therefore$ (CC) Las experiencias sensoriales tienen contenido conceptual. (Brewer, 2005, p. 218).

Con la expresión "creencias empíricas" Brewer $(1999$, p. 19) hace referencia a lo que aquí denominamos 'CEPs', es decir, a nuestras creencias de experiencia más básicas. De ellas afirma (Brewer, 1999 p. xiv; pp. 18 y ss.) que obtienen su contenido en virtud del hecho de estar en ciertas relaciones con las EPs. Pero añade que sólo la relacion de 'proporcionar razones' o 'dar razón de' entre EPs y CEPs posibilita determinar el contenido empírico de estas últimas; de no ser así quedaría excluida, según él, cualquier posibilidad de tener creencias acerca del entorno inmediato extra-mental. Si esto es cierto, y puesto que es un hecho que poseemos este tipo de creencias, (1) ha de ser necesariamente verdadera. Ahora bien, ¿qué entiende exactamente este autor por 'proporcionar razones'?

Podemos encontrar una respuesta al exponer la argumentación que lleva a cabo como defensa de la premisa (2). Brewer infiere ésta a partir de dos subpremisas con las que pretende plasmar las condiciones que, según él, ha de cumplir todo aquello que se erija como razón:

(2A) dar razones implica identificar ciertas proposiciones relevantes que figuren como premisas y conclusión en un argumento válido.

(2B) esas razones han de ser razones propias del sujeto, aquellas que él mismo puede reconocer como tales razones.

(Brewer, 1999, pp. 150 y ss.; 2005, pp. 218-219).

Con (2A) quiere resaltar una íntima conexión entre razones e inferencias. Cuando intentamos dar razón de algo ponemos de manifiesto un tipo de aprobación que puede ser explicitada por medio de un argumento. Por ello, Brewer entiende que el contenido de lo que se ofrece como razón debe ser tal que pueda formar parte de una inferencia de algún tipo en calidad de premisa, es decir, que pueda ser integrable en un argumento válido mediante el que se infiera como conclusión la creencia que se quiere justificar. ${ }^{11}$ Puesto que consideramos la proposición como el

11 Aunque no está muy claro en el discurso de Brewer, interpretamos que no se trata obviamente de que el razonamiento en cuestión deba hacerse efectivo en toda ocasión sino sólo que ello sea posible. 
tipo de entidad susceptible de conformar inferencias, el contenido de cualquier estado mental que se ofrezca como la razón de una creencia debería poseer, según Brewer, una naturaleza proposicional.

En 2B adopta una postura internalista, manifiesta también en los planteamientos de McDowell, en referencia a la justificación de CEPs. Al explicar dicha justificación, Brewer considera que se ha de dar cuenta de la posibilidad de que el agente de la misma sea el propio sujeto. Por tanto, las razones a tener en cuenta han de ser las propias del sujeto, sus razones, aquellas que figuren desde su punto de vista en el sentido de que él mismo, en primera persona, pueda reconocerlas como tales razones. El caso es que para Brewer ese reconocimiento por parte del sujeto sólo es posible si existe una relación de identidad entre el contenido del estado perceptual que experimenta y el contenido de la proposición con la que caracteriza dicho contenido perceptual, proposición que explicitaría como premisa en una inferencia mediante la que concluiría la creencia que desea justificar. Esa identidad implica que el contenido perceptual en cuestión no sólo es proposicional sino que está estructurado por los conceptos que el sujeto utiliza al caracterizarlo. Brewer considera que sólo de ese modo se garantiza que el sujeto comprende el contenido de su EP y lo reconoce como su razón para creer. De no ser así no habría forma de garantizar que tal reconocimiento se produce. Para Brewer (1999, pp. 161-162) el noconceptualista se ve abocado a ofrecer una explicación de corte fiabilista que dejaría fuera el punto de vista del sujeto. La única alternativa que le quedaría para poder dar cuenta de ese punto de vista sería, según Brewer (1999, p. 168), considerar que el sujeto realiza dicho reconocimiento por la vía de apelar a un conocimiento de segundo orden relativo a la relación existente entre el tipo de EPs como las que está teniendo -con ese contenido supuestamente no-conceptual- y la verdad de creencias del tipo de la que quiere justificar. Así, la justificación de su creencia implicaría un razonamiento con un esquema similar al siguiente:

(i) Este estado (una determinada EP) es F

(ii) Cualquiera cosa que sea $\mathrm{F}$ es una razón para creer que $\mathrm{p}$.

(iii) Por lo tanto, tengo una razón para creer que $\mathrm{p}$.

(ii) encierra ese conocimiento de segundo orden al que alude. Pero obviamente ésta no es la forma habitual de reconocer nuestras EPs como razón de nuestras creencias de experiencia más básicas. En situaciones corrientes no parece adecuado suponer que el sujeto realice un ejercicio reflexivo de este tipo. Brewer (1999, p. 169) estima que en caso de aceptar esta forma indirecta de reconocimiento, la verdadera razón para creer residiría en último término en ese conocimiento de segundo orden y no ya en el contenido perceptual mismo, lo cual significa para él una constatación más de que todo contenido que funcione como razón ha de ser conceptual. 
Queda claro, en definitiva, cómo hemos de interpretar la expresión "proporcionan razones" en el argumento de Brewer: las EPs proporcionan razones porque sus contenidos son de tal modo que pueden erigirse en una suerte de premisas integrables en inferencias que el propio sujeto perceptor puede realizar. Puesto que sólo estados mentales conceptuales pueden cumplir todos los requerimientos explicitados en $2 \mathrm{~A}$ y $2 \mathrm{~B}$, Brewer concluye que las EPs proporcionan razones porque su contenido es conceptual.

3. ¿Hemos de admitir entonces que la posesión de contenido conceptual es condición necesaria para que las EPs jueguen algún rol epistémico normativo? Nosotros creemos que no, y que tras este tipo de argumentación se esconde una concepción general de 'contenido' que se nos antoja sumamente controvertida. Se trata de lo que Bermúdez (1998, pp. 41-43) denomina "concepción clásica del contenido", según la cual lo que se puede atribuir como contenido a un sujeto depende de las capacidades conceptuales de ese sujeto, las cuales se entiende que a su vez están estrechamente ligadas a sus capacidades lingüísticas ${ }^{12}$. En los siguientes pasajes se pone de manifiesto la adhesión de McDowell a esta concepción:

If content is dualistically opposed to what is conceptual, 'content' cannot mean what it often means in contemporary philosophy, namely, what is given by a "that" clause in, for instance, an attribution of a belief (McDowell, 1994a, p. 3);

If experience is pictured as input to spontaneity from outside, then it is another case of fraudulent labelling to use the word "content" for something we can even so take experience to have, in such way that reason-constituting relations can intelligibly hold between experiences and judgements. (McDowell, 1994a, p. 53).

Del primero se colige que McDowell entiende que la experiencia perceptual, en tanto que poseedora de contenido, no es sino un tipo de actitud proposicional, y que el ofrecer su contenido, al igual que en el caso de la creencia, no consiste en otra cosa que en especificar la proposición hacia la que el sujeto adopta esa actitud. En el segundo vemos que para McDowell el mero hecho de concebir un contenido como no conceptual supone falsear la noción misma de 'contenido'. No es de extrañar pues que para McDowell y Brewer sólo se puedan establecer relaciones racio-

12 Bermúdez (1998) presenta esta concepción como basada en los siguientes principios: 'Principio del requisito conceptual' [Conceptual Requirement Principle] y 'Principio de prioridad' [Priority Principle]. Conforme al primero, el rango de contenidos que se puede atribuir a una criatura está directamente determinado por los conceptos que esa criatura posee. Conforme al segundo, las habilidades conceptuales están constitutivamente ligadas a las habilidades lingüísticas de un modo que resulta imposible que sean poseídas por criaturas carentes de lenguaje. Bermúdez rechaza el primer principio aunque acepta el segundo. 
nales entre contenidos conceptuales. Teniendo esto en cuenta se puede afirmar que una primera consecuencia de su adopción de esta concepción clásica de contenido es que los dos coinciden en subrayar que cualquier interpretación normativa del rol de apoyo epistémico, incluso si tal apoyo es ejercido por una EP, debería acomodarse en general al paradigma del razonamiento o construcción de inferencias. En efecto, resulta patente que ambos consideran la justificación inferencial entre creencias como el modelo relevante a seguir para dar cuenta del apoyo epistémico normativo que las EPs proporcionan a las CEPsi3.

Otra consecuencia de adoptar la concepción clásica de contenido tiene que ver con el carácter representacional de la experiencia perceptual. Teniendo en cuenta que suele asumirse que los estados mentales representacionales poseen contenido en tanto que representan de un cierto modo determinados estados de cosas, el conceptualista se ve comprometido a defender que ese modo perceptual de representar el mundo (el contenido representacional de las EPs) estará necesariamente determinado por las capacidades conceptuales del sujeto de dichos estados mentales. Siendo así, es la posesión de determinados conceptos lo que permite al sujeto perceptor apreciar el modo en el que se le presenta un determinado estado de cosas. Por ello McDowell y Brewer dan a entender continuamente que un contenido perceptual no-conceptual no podría resultar epistémicamente significativo o inteligible para un sujeto (humano y adulto). Las EPs con contenido no-conceptual no serían más que intuiciones ciegas, opacas en el sentido de que el sujeto perceptor no gozaría de una adecuada comprensión de su contenido, de que cognitivamente no serían nada para él.

Una forma diferente de entender esa supuesta opacidad de los contenidos noconceptuales sería decir que el sujeto no tendría consciencia de, ni acceso cognitivo a, tales contenidos. En tal caso estaríamos caracterizándolos como contenidos de estados mentales situados a un nivel subpersonal. Se entiende que el ámbito de lo subpersonal abarca aquellas operaciones que no pueden ser atribuibles a personas en tanto que conscientes, sino a sistemas parciales o modulares de procesamiento de información, y por lo tanto fuera del alcance de la consciencia y el acceso cognitivo del sujeto. Pues bien, al menos McDowell (1994a, p. 55) parece considerar que, en último término, hablar de contenidos no-conceptuales sólo sería legítimo al referirnos a estados subpersonales, y ello únicamente asumiendo que en ese caso se

\footnotetext{
13 Estamos convencidos de que esta interpretación de las consideraciones de McDowell (1994a) es apropiada, además de ser la habitual, y ello a pesar de que más recientemente, tal y como adelantamos en la nota 4, él mismo (2009, p. 270) haya negado que en aquella obra presentara el contenido perceptual como una premisa o a la justificación que dicho contenido permite como una suerte de inferencia. En el caso de Brewer no cabe una interpretación errónea, pues señala explícitamente (1999, p. 166) que el razonamiento deductivo constituye el modelo en el que poder hablar de razones en un sentido genuino.
} 
está usando el término "contenido" como una mera forma de expresarse, como una analogía o sencillamente como una herramienta explicativa14.

Convenimos con Bermúdez en rechazar semejante concepción general de 'contenido', así como las restricciones que tal concepción conlleva. Bermúdez (1995) defiende la legitimidad de hablar de 'contenidos no-conceptuales' tanto en el ámbito de la percepción consciente como en el ámbito de lo subpersonal toda vez que quepa entender que la propiedad de ser representacional puede ser satisfecha con independencia de la posesión de conceptos. A este respecto Bermúdez (1995, p. 200) sugiere una serie de criterios que considera suficientes para determinar si un estado mental es representacional: a) que permita explicar conductas que no son mecánicas; b) que pueda integrarse cognitivamente o interactuar con otros estados mentales; c) que presente algún tipo de estructura o de composicionalidad15; y e) que quepa la posibilidad de que represente erróneamente. Estos criterios deberían poder ser cumplidos con independencia del tipo de contenido (conceptual o no) que se le atribuya a dicho estado. Desde esta perspectiva cabría entonces la posibilidad de considerar las EPs como estados mentales representacionales aun poseyendo contenido no-conceptual.

Siendo factible lo que Bermúdez sugiere, pensamos, en primer lugar, que sería legítimo hablar de contenidos no-conceptuales a nivel personal (de hecho, en la segunda sección de este trabajo defenderemos que hay razones para considerar la existencia de representaciones perceptuales no-conceptuales perfectamente personales). En segundo lugar, entendemos que no habría motivos suficientes para negar que un contenido perceptual no-conceptual resulte significativo e inteligible para el sujeto perceptor. Es más, pensamos que en su afán por ofrecer una propuesta de corte kantiano tanto Brewer como McDowell se han excedido en su interpretación del aforismo de la Crítica de la Razón Pura según el cual las intuiciones sin conceptos son ciegas, y entendemos que hay lugar para interpretaciones de dicho aforismo menos radicales y más compatibles con una posición no-conceptualista ${ }^{16}$. En tercer lugar, y por último, la solución de Bermúdez nos induce a creer que tampoco habría motivos suficientes, en contra de lo que pretende el conceptualista, que nos impidan pensar en una relación epistémica normativa entre dos contenidos

\footnotetext{
14 Debemos señalar que en este ámbito McDowell (1994b, p. 198) excluye cualquier posibilidad de hablar de contenido representacional al entender que los sistemas subpersonales son mecanismos físicos, ingenios exclusivamente sintácticos, no semánticos.

15 Como veremos más adelante, el tipo de composicionalidad que muestran las EPs, en tanto que noconceptuales, ha de diferir necesariamente de la exhibida por contenidos conceptuales.

16 De hecho, Hanna (2005, pp. 256-257) señala que lo que Kant quiere decir cuando habla de "intuiciones ciegas" es sencillamente que por sí solas, sin la participación del entendimiento, no darían lugar a juicios de experiencia objetivamente válidos. Pero que ello no significa que Kant entienda que las intuiciones en sí mismas estén absolutamente vacías de significación o no puedan ser entendidas como cognitiva y semánticamente independientes.
} 
(representacionales) aun cuando tal relación se establezca desde uno no-conceptual a uno conceptual 17 , y sin necesidad de interpretarla conforme al modelo de la justificación inferencial entre creencias. Nuestra pretensión es, de hecho, ofrecer una propuesta basada en términos de lo que llamaremos 'sustento epistémico', entendido éste como algo diferente de lo que tradicionalmente se ha entendido por 'justificación de creencias perceptuales'. Para ello, como ya anunciamos, partimos de algunos planteamientos de Fodor.

\section{Fodor y las representaciones mentales no-conceptuales}

1. Fodor (2007, pp. 111 y ss.; 2008, pp. 185 y ss.) señala que existe evidencia empírica de la existencia de representaciones mentales no-conceptuales en el proceso de percepción. Defiende asimismo que tales representaciones pueden justificar creencias y no ser sólo mera causa de ellas. Estas afirmaciones podrían considerarse un espaldarazo para un enfoque no-conceptualista sobre el papel epistémico de las EPs. Ahora bien, su atractivo se desvanece parcialmente cuando vemos que al mismo tiempo defiende que dichas representaciones se dan en niveles tempranos del procesamiento de la información perceptual, y que por ello han de concebirse como subpersonales (Fodor, 2007, p. 106).18 Siendo así, quedan fuera del alcance de la consciencia e introspección del sujeto, lo cual, a nuestro entender, hace incomprensible que puedan ejercer un papel epistémico normativo. En lo que sigue revisaremos este aspecto de su planteamiento y trataremos de mostrar que tales representaciones perceptuales no-conceptuales se dan a nivel personal.

Fodor (2007, pp. 107 y ss.; 2008, pp.170 y ss.) tipifica esas representaciones dentro del ámbito de lo que denomina 'representaciones icónicas' (en adelante RIs), ámbito al que también pertenecen cosas tales como pinturas, fotos, gráficos, etc. A este tipo de representaciones contrapone lo que serían 'representaciones discursivas' (en adelante RDs), entre las cuales las oraciones de los lenguajes naturales se presentan como paradigma. Las RIs son no-conceptuales o precategoriales, mientras que las RDs son claramente conceptuales. En tanto que representaciones,

\footnotetext{
17 Esto estaría en concordancia con la apreciación ya mantenida por Heck (2000, pp. 504 y ss.) de que cabría pensar en el carácter representacional del contenido perceptual como la verdadera precondición o condición necesaria, aunque no suficiente según él, para poder responder afirmativamente a la cuestión de si nuestras EPs justifican CEPs. Así, según Heck, (2000, p. 505) en vez de interpretar el espacio de las razones como incluido en el ámbito de lo conceptual, deberíamos más bien interpretarlo como «incluido dentro del espacio del contenido representacional». Nosotros defenderemos algo ligeramente diferente (parte III de este trabajo).

18 Téngase en cuenta que, conforme a su teoría modular de la mente, los procesamientos de información a niveles tempranos se desarrollan dentro de sistemas de entrada o módulos encapsulados, sin influencia de, ni acceso a, representaciones de los sistemas centrales.
} 
ambos tipos son evaluables, es decir, todas son correctas o incorrectas dependiendo de si son o no apropiadas en relación a su objeto de representación. Fodor señala dos criterios para distinguirlas: el tipo de composicionalidad semántica que exhiben y el hecho de que permitan, o no, individuar en lo representado. 19

Toda representación posee composicionalidad semántica porque su contenido semántico está determinado por el contenido semántico de sus constituyentes, cada uno de los cuales representa de forma autónoma una parte de lo representado. Ahora bien, según Fodor, en el caso de las RDs existe un modo canónico de descomponerlas que fija qué partes de dichas representaciones son constituyentes semánticos y cuáles no. Así, por ejemplo, en una oración como "Mi gato está dormido en el sofá" fijaríamos como constituyentes semánticos las expresiones "Mi gato", "gato", "está dormido", "en el sofá", "el sofá", o "está en el sofá", pero nunca "Mi ... está" o "gato ... el". 20 Por su parte las RI no se descomponen canónicamente, según Fodor, sino siguiendo lo que llama 'Principio Pintura':

$P P$ : "If $\mathrm{P}$ is a picture of $\mathrm{X}$, then parts of $\mathrm{P}$ are pictures of parts of $\mathrm{X}$ " (Fodor, 2007, $\mathrm{p}$. 108).

Conforme a esto, todas las partes resultantes serían a su vez RIs de partes del objeto o estado de cosas que esa RI representa, lo cual hace de esta descomposición algo análogo a la descomposición de un rompecabezas en sus piezas componentes. La composicionalidad semántica está igualmente asegurada ya se aplique una descomposición canónica o el $P P$, pero la peculiaridad de éste estriba en que permite formas arbitrarias de descomponerse en partes que, según Fodor, son a su vez representacionales. 21

Más interesante incluso es el otro aspecto en el que RDs y RIs se diferencian; a saber: la capacidad o incapacidad de establecer individuaciones en lo representado. Una RD proporciona una manera de individuar elementos de lo representado y así distinguir unos de otros, mientras que una RI sólo permite discriminar aspectos de lo representado. Discriminar aspectos de lo representado no es lo mismo que distinguir individuos en lo representado. La individuación se hace posible gracias al aparato conceptual que una $\mathrm{RD}$ entraña y del cual carecen las RIs. Fodor plantea que las RDs, en tanto que permiten individuar, se ven afectadas por lo que denomina 'efecto item', consistente en que su grado de "complejidad psicológica" -por ejem-

19 Balog (2009) proporciona un análisis crítico de estas propuestas de Fodor.

20 El ejemplo es nuestro.

21 No es obvio que cualquier forma de descomponer nos provea de partes que representen algo de forma autónoma. Pensemos, por ejemplo, en puntos o pixeles de un icono determinado o, sin ir tan lejos, en las pequeñas piezas de un mosaico. En todo caso lo que Fodor realmente precisa es solamente que las RIs no posean un modo canónico de descomposición. 
plo, la cantidad de memoria que se requiere para almacenar o procesar la información que proporcionan - varía en función del número de individuos cuyas propiedades especifica. Así, la cantidad de memoria requerida para procesar la información de, por ejemplo, el listín telefónico de una gran ciudad, es mayor que la necesaria en el caso de una pequeña ciudad de provincias. Nada de esto ocurre con una RI. Procesar la información que suministra, por ejemplo, una foto de grupo, no implica una mayor cantidad de memoria que en el caso de una foto de pareja. El efecto item se puede utilizar pues como un criterio para dirimir si estamos ante una RD, y por ello conceptual, o ante una RI, cuyo contenido hemos de considerar no-conceptual.

2. Fodor considera dos experimentos que, según él, ponen en evidencia la existencia de representaciones mentales que exhiben los efectos típicos de RIs. Uno de ellos, en el que nos centraremos, es el de Sperling (1960) sobre las representaciones de la memoria icónica (en adelante RMIs). ${ }^{22}$ En su versión original los sujetos del experimento eran expuestos a presentaciones taquistoscópicas de una serie de matrices o mosaicos de letras, algunas compuestas de tres filas con cuatro letras por fila, otras de un número mayor. La presentación de cada una de ellas no superaba los $50 \mathrm{~ms}$ e iban seguidas siempre de un campo en blanco. Proyectarlas mediante un taquistoscópio aseguraba una única fijación ocular en el campo visual por cada presentación, a partir de la cual los participantes debían identificar el mayor número de letras posible. Este planteamiento fue denominado "informe total" y el resultado fue que los participantes sólo lograban recordar entre 4 y 5 letras, con independencia de cuantas $(9,12$, o más) configurasen la matriz, aunque curiosamente afirmaban tener la sensación de haber visto más letras de las que lograban identificar.

Para comprobar si tan exiguo rendimiento se debía a la extrema brevedad de las presentaciones se fue variando su duración entre 15 y $500 \mathrm{~ms}$, pero el resultado fue el mismo. Sperling propuso entonces una explicación alternativa sugiriendo que la totalidad del conjunto estimular era sensorialmente captada dejando una huella de memoria de muy escasa duración. Esa huella de memoria, o 'memoria icónica', se desvanecería en el breve espacio de tiempo entre el final de la presentación y la producción de la respuesta verbal o identificación, aunque debería ser suficiente para explicar el hecho de que a los sujetos participantes les pareciese haber percibido más letras que las que lograron identificar. Con el fin de corroborar esta hipótesis de la huella de memoria Sperling empleó, en contrapartida al informe total, la llamada "técnica del informe parcial", consistente en requerir de los participantes que identificasen las letras de una sola de las filas de la matriz en función de un determinado tono auditivo. Para ello se hizo corresponder un tono auditivo alto para la fila superior, uno medio para la fila media y uno bajo para la inferior. Los sujetos

22 Crespo (2006, pp. 243-253) nos brinda una breve panorámica sobre este experimento y sus posteriores variantes. 
no sabían a qué fila pertenecerían las letras a identificar hasta que, coincidiendo con el final de la presentación de la matriz, se hacía sonar uno de los tonos elegido al azar. El resultado era que identificaban una media de tres de las cuatro letras de cada fila. Se obtenía así una mejora de rendimiento que parecía ratificar la existencia de la citada huella de memoria. Posteriormente se intentó comprobar su duración media introduciendo en el informe parcial demoras de la señal auditiva con respecto a la desaparición de la matriz. Se constataron mermas de rendimiento proporcionales a dichas demoras, hasta el punto de que cuando la señal se demoraba un segundo el rendimiento resultaba equiparable al obtenido mediante el informe total, lo cual indicaba que la huella se desvanece en un brevísimo espacio de tiempo. De hecho, suele aceptarse que su duración media oscilaría entre 500 y $800 \mathrm{~ms}$ (Crespo, 2006, p. 252), muy breve pero suficiente para que los sujetos experimenten haber tenido presente la totalidad de la matriz.

Fodor (2008, p. 189) señala que los resultados de una variante experimental de la técnica de informe parcial dan lugar a considerar que estas representaciones de la memoria icónica son efectivamente no-conceptuales. A diferencia de lo que sucedía en la versión original, en la que la señal auditiva indicaba una posición (la fila que se debía recordar), esta vez se presentan matrices que combinan letras con números, y las señales auditivas indican unas veces la fila y otras la categoría (números o letras) que se ha de recordar. El resultado es que el rendimiento disminuye notablemente cuando el tono elegido indica que los participantes deben identificar sólo letras o sólo números (Crespo, 2006, pp. 252-253). La introducción de las categorías 'letra' y 'número' no tiene pues una influencia positiva, sino más bien negativa, sobre el rendimiento que cabría esperar tratándose de un informe parcial. De ello se puede concluir que los contenidos de la memoria icónica son precategoriales o se producen con independencia de los conceptos o categorías que posea un sujeto.

3. Fodor ve en las huellas de memoria icónica unos firmes candidatos a RMIs. Cabe apuntar, no obstante, que la introducción de este tipo de representaciones supone una novedad dentro del planteamiento general de Fodor respecto al proceso de percepción, puesto que este autor tradicionalmente ha considerado las representaciones implicadas en ese proceso como conceptuales. Así, en Fodor (1983, III.6) el carácter conceptual de las EPs se debía a que los sistemas de entrada encargados del análisis de la información sensorial categorizan los estímulos mediante un tipo peculiar de conceptos que denominaba "categorías básicas". Ahora, en Fodor (2007; 2008), la presencia de representaciones no-conceptuales (las RMIs) en fases tempranas del procesamiento -en concreto, inmediatamente después de las transducciones- contrasta con el carácter conceptual que conservan los productos finales de ese proceso: las representaciones de la experiencia fenoménica (la experiencia perceptual). Este carácter conceptual se debe, en los últimos planteamientos de 
Fodor, al hecho de que los procesos que se dan en fases más cercanas a la conformación final de la experiencia fenoménica son, según él, procesos inferenciales. ${ }^{23}$

Pese al carácter no-conceptual de una RMI, Fodor (2007, pp. 114-115; 2008, pp. 193-194) defiende que su disponibilidad previa proporciona una razón para alcanzar alguna creencia adecuada. Así, frente a los posicionamientos conceptualistas, entiende que el hecho de que la justificación sea una relación entre contenidos de representaciones no implica que lo sea entre contenidos exclusivamente conceptuales. Sin embargo, por nuestra parte creemos que el carácter subpersonal que atribuye a esas RMIs impide establecer esa relación de justificación o cualquier otra relación epistémica normativa. No comprendemos cómo un sujeto podría establecer una relación de esa índole entre contenidos no-conceptuales y contenidos conceptuales si los primeros no resultan accesibles a su consciencia. Por ello trataremos de mostrar a continuación que, pese a Fodor, hay razones para considerar que las RMI son personales. 24

Nuestra experiencia fenoménica de la percepción visual es la de un flujo continuo. No obstante, una serie de experimentos muestran que en realidad captamos la información visual de una forma discreta. En efecto, resulta que la captación de información se produce sólo en los momentos de fijación ocular, en los que el sujeto consigue focalizar su atención en una parte de la escena. Pero estas fijaciones duran brevísimos periodos de tiempo (entre 175 y $500 \mathrm{~ms}$ ) y cada una de ellas va seguida por un movimiento ocular muy rápido, llamado "sacádico", que si bien sirve para que el sujeto dirija su atención a otra parte de la escena, provoca que durante el mismo no se produzca captación alguna de información (fenómeno llamado 'supresión sacádica'). Puesto que entre dos fijaciones oculares consecutivas se produce uno de estos movimientos sacádicos, se genera entonces una discontinuidad en la captación de información que contrasta con la mencionada continuidad fenoménica de nuestra experiencia visual. Esta continuidad debe estar asegurada entonces por algún mecanismo que permita integrar todas esas captaciones discontinuas de forma que las experimentemos como un flujo continuo. Pues bien, se ha propuesto la hipótesis, inicialmente formulada por Coltheart (1983), de que son las RMIs las que constituyen ese elemento integrador. La razón estriba en que una RMI perdura el tiempo suficiente como para cubrir el paso de una fijación ocular a otra a través de un sacádico. Así, secuencias de RMIs relativas a una escena determinada estarían conformando la apariencia o experiencia fenoménica visual de esa

\footnotetext{
23 Se trata de inferencias subpersonales, entre las que incluye las operaciones que dan lugar a constancias perceptuales de forma, tamaño, color, etc. (Fodor, 2007, p. 114; 2008, p. 192).

${ }^{24}$ Estamos de acuerdo con Heck (2007, p. 119) en su apreciación de que de ser subpersonales las RMIs postuladas por Fodor, no tendrían la relevancia epistemológica suficiente como para suponer un desafío a la posición conceptualista. No obstante, Heck, a diferencia de lo que nosotros hacemos a continuación, no llega a proporcionar ninguna razón en concreto que permita la caracterización de las RMIs como personales.
} 
escena. ${ }^{25}$ La hipótesis en cuestión hace entonces que resulte mucho más plausible la idea de considerar a esas RMIs a un nivel personal, y no, como defiende Fodor, a un nivel subpersonal.

\section{Sustento epistémico}

1. Una vez salvado el inconveniente que supondría entender las RMIs como subpersonales ya podemos ofrecer una propuesta en positivo del modo en que las representaciones de la experiencia perceptual, conformadas por esas RMIs no-conceptuales, constituyen un sustento epistémico para juicios y creencias perceptuales. Para ello volvemos a Fodor, esta vez retrotrayéndonos a "Observation Reconsidered”, de 1984 (pp. 40-41). Allí establece una clara distinción entre 'fijación de apariencias' y 'fijación de creencias perceptuales'. La diferencia consiste en que la fijación de apariencias o representaciones fenoménicas, aun siendo un proceso inferencial, no se ve afectada por todo el bagaje de creencias disponible por el sujeto, mientras que la fijación de CEPs sí se ve afectada. Según Fodor, en la fijación de apariencias sólo opera un subconjunto de creencias y además de manera autónoma en el módulo que conforma el sistema visual. Se trata por lo tanto de un proceso encapsulado. El resultado de ese proceso, y esto es lo que aquí nos interesa, consiste para Fodor en representaciones a modo de meras hipótesis acerca de las fuentes distales de la estimulación. ${ }^{26}$ Dichas hipótesis son lo que un sujeto llegaría a creer acerca de las fuentes distales si en la consiguiente fijación de creencias sólo intervinieran las apariencias o EPs relevantes. Pero lo que sucede en la fijación de creencias es, según Fodor, que cada una de las EPs del sujeto, en tanto que hipótesis, son sometidas a los procesos centrales para ser evaluadas a la luz de todo el bagaje de creencias de ese sujeto. Como resultado de esa evaluación el sujeto puede formarse una o varias creencias acerca de su entorno inmediato.

Al igual que Fodor nosotros también postulamos este tipo de hipótesis; pero con la diferencia de que no se corresponderían con las EPs mismas, dado el carácter noconceptual que atribuimos a estas últimas. Más bien, entendemos que tales hipótesis se obtienen a partir de las EPs en función del aparato conceptual disponible por el sujeto de esas experiencias y como resultado de atender y seleccionar determi-

\footnotetext{
25 Cabe admitir que se trata de una hipótesis controvertida. En concreto, se ha considerado que los casos de ceguera al cambio y de ceguera atencional constituyen objeciones a la misma (Crespo, 2006, pp. 269 y ss.). Sin embargo, recientemente se han llevado a cabo otros experimentos que lejos de refutarla proporcionarían claves para su comprensión. Entre los trabajos que apuntan en tal dirección están los de Hayhoe et. al. (1998), Fernández-Duque et. al. (2000) y Becker et. al. (2000).

26 Quizás sea ese carácter de hipótesis la razón por la que Fodor considera que las EPs resultan inadecuadas para sustentar CEPs y haya decidido buscar ese sustento en fases más tempranas del procesamiento de la información visual.
} 
nados aspectos de su contenido informativo. Así, aunque el contenido de una EP no sea conceptual, el de las hipótesis a ella asociadas sí lo es. Las hipótesis contempladas por un sujeto en relación con cierta EP se someterían, como ya planteaba Fodor, a los procesos centrales para ser evaluadas a la luz de todo el bagaje de conocimientos y creencias disponibles por el sujeto, fijando así una determinada CEP.

Descrito con más detalle el proceso es el siguiente. Un sujeto $\mathrm{S}$ tiene cierta EP (o una secuencia de EPs) de una escena E. Esa(s) EP(s) puede desencadenar en S ciertas hipótesis (al menos una) que podrían tomarse como posibles descripciones de E. Llamémosles Hipótesis Perceptuales Directas [HPDs]. Estas HPDs se desencadenan como resultado de: (i) atender y seleccionar determinados aspectos del contenido informativo de la(s) EP(s); (ii) en función del aparato conceptual del que $\mathrm{S}$ dispone; y (iii) en función del aprendizaje perceptual de $\mathrm{S}$ en relación con EPs previas que sean similares para $\mathrm{S}$. $\mathrm{La}(\mathrm{s}) \mathrm{HPD}(\mathrm{s})$ desencadenada(s) por aquella(s) EP(s) de S se someterían, como ya planteaba Fodor, a los procesos centrales para ser evaluadas a la luz de todo el bagaje de creencias disponibles por parte de $\mathrm{S}$, fijando así una(s) determinada(s) CEP(s) de S. Si la(s) EP(s) sólo desencadena(n) una HPD, ésta será o no adoptada por $\mathrm{S}$ como CEP suya dependiendo de si entra o no en colisión con el bagaje de fondo. Si desencadena varias HPDs, de entre ellas el sujeto adoptará como su(s) CEP(s) alguna(s) de aquellas HPDs que no entra(n) en colisión consciente con las creencias ya asumidas por ese sujeto. Si todas las HPDs desencadenadas inicialmente entran en colisión consciente con las creencias asumidas por S, éste se ve impelido a contemplar alguna otra alternativa que pueda adoptar como creencia acerca de la EP en cuestión. De este modo, el bagaje de creencias de $\mathrm{S}$ puede dar lugar a desarrollar otra(s) hipótesis perceptual(es) no-directa(s) [HPND(s)] alternativa(s) a la(s) HPD(s), tal que el contenido de la CEP fijada sea el contenido de esa (o una de esas) hipótesis alternativa(s). Claro que, normalmente, y a falta de variaciones en las creencias relevantes asumidas por un sujeto, la hipótesis que éste puede contemplar y adoptar como CEP vendrá prefijada por el aprendizaje a partir de EPs similares previas. No obstante, la posibilidad de que la creencia adoptada acerca de una EP no corresponda a una HPD, sino a una HPND desarrollada conforme a las creencias ya asumidas, no conlleva que la propia EP pueda variar en función de las creencias asumidas.

Ilustremos esto con un ejemplo. Supongamos un sujeto $\mathrm{S}$ ante un racimo de uvas de cera indiferenciable a primera vista para $\mathrm{S}$ de un auténtico racimo de uvas. En este caso S tendría la misma EP27 (no conceptual) tanto si (i) fija la CEP de que "es un racimo de uvas" como si (ii) fija la CEP de que "es un racimo de uvas de cera" una vez que ha verificado, por ejemplo a través del tacto, que no se trata de un racimo de uvas auténtico. En (i), cuando S fija la CEP de que "es un racimo de

\footnotetext{
27 Podría ser, como ya se indicó, una secuencia de EPs de cierta escena.
} 
uvas", su EP visual habría propiciado al menos la HPD de que "es un racimo de uvas". En (ii), cuando S fija la creencia de que "es un racimo de uvas de cera", cabrían las siguientes alternativas:

a) su EP visual desencadena dos HPDs: "es un racimo de uvas" y "es un racimo de uvas de cera";

b) su EP visual desencadena simplemente la HPD "es un racimo de uvas" y $\mathrm{S}$ obtiene gracias a su bagaje de creencias la HPND "es un racimo de uvas de cera";

c) su EP visual simplemente desencadena la HPD "es un racimo de uvas de cera" gracias al aprendizaje visual previo que $\mathrm{S}$ es capaz de poner en juego.

En cualquiera de los casos la fijación de la creencia requiere que mediante la participación de los procesos centrales $\mathrm{S}$ elija la hipótesis más apropiada a la luz de su bagaje de creencias.

Este planteamiento permite entender también los casos de ilusión óptica. Pensemos por ejemplo en la ilusión de Müller-Lyer. Nuestra propuesta proporciona un marco adecuado para explicar de forma integrada por qué cuando $\mathrm{S}$ percibe las dos líneas paralelas:

a) persiste la ilusión -es decir, la EP (o secuencia de EPs)- aun cuando S conoce que se trata de una ilusión;

b) S contempla a modo de hipótesis la consideración conceptual ilusoria de que una de las líneas es más larga que otra;

c) S fija la creencia (correcta) de que las líneas son de la misma longitud a partir de una EP que le muestra lo contrario.

Según lo señalado, podemos decir que una EP (o secuencia de EPs) de un sujeto $S$ acerca de una escena $E$ es el sustento epistémico de una determinada CEP de $S$ acerca de E en la medida en que el contenido de esa CEP (falible) es el contenido de la mejor hipótesis a disposición de S, entre sus HPDs y (de ser el caso) sus $H P N D$ s correspondientes a esa EP (o secuencia de EPS), para dar cuenta descriptivamente de $E$. La fijación de CEPs se alcanza mediante una suerte de inferencia a la mejor explicación o de inferencia a lo que mejor da cuenta descriptivamente de cómo son o acontecen las cosas alrededor de $\mathrm{S}$, dado el bagaje de creencias relevantes asumidas por S. El sentido en el que ahora hablamos de que una EP sustenta una CEP no se corresponde con nada de lo que en la tradición empirista se ha entendido por justificación de CEPs. Una EP (o una secuencia de EPs) mantiene una relación epistémica normativa de sustento con una CEP debido a que una (o varias) HPD(s), desencadenada(s) por esa(s) EP(s), es (son) sometida(s) a evaluación a la 
luz del bagaje de creencias asumidas, y de manera específica con el bagaje relevante a esa(s) HPD(s).

2. ¿Cuál es la peculiaridad de nuestra propuesta? En relación al debate entre las posiciones conceptualistas de McDowell (1994) y Brewer $(1999 ; 2005)$ y las noconceptualistas de Evans (1982) y Peacocke $(1992 ; 2001)^{28}$ ha quedado claro que a diferencia de las primeras lo que planteamos no se ajusta propiamente al modelo de justificación inferencial entre creencias. El rol que atribuimos a las EPs no implica entender su contenido como una suerte de premisa para una suerte de argumento o inferencia. Pero nuestra propuesta, aun siendo no-conceptualista, se diferencia también de las segundas si tenemos en cuenta que también en ellas se ha considerado que el papel epistémico de las EPs estriba en que en sí mismas constituyen las razones del sujeto para creer, si bien negando que el carácter conceptual de las EPs sea un requisito para ello. A nuestro modo de ver las EPs no juegan el papel de constituirse en razones para creer sino el de permitir, o contribuir a, el establecimiento de razones para creer.

En este sentido lo que proponemos se aproxima a planteamientos como los de Burge (2003), Peacocke (2004) y Pryor (2005). En esos trabajos se mantiene que el contenido de las EPs no es proposicional y que éstas mantienen con las correspondientes CEPs un tipo de relación epistémica (un tipo de garantía epistémica, según Burge) distinto del que corresponde a la justificación en base a razones. ${ }^{29}$ Aunque para denominar ese tipo de relación epistémica Burge y Peacocke (2004) hablan de "legitimación" y Pryor de "justificación inmediata (o no inferencial)", con el fin de simplificar consideraremos que ambas expresiones son equivalentes y hablaremos de legitimación. ${ }^{30}$ La relación de legitimación no conlleva, según ellos, que el suje-

28 También Heck (2000).

29 Burge habla de dos tipos de garantía [warrant] epistémica: justificación y legitimación; mientras Pryor habla de dos tipos de justificación: mediata e inmediata. Por su parte algunos pasajes de Peacocke (2004) dan a entender que éste incluye las legitimaciones como una manera de dar razones para creer, tanto por lo que dice de la legitimación de CEPs por EPs (que él parece hacer caer en el ámbito de dar razones), como por considerar que la relación de legitimación se da cuando un sujeto se auto-adscribe creencias sobre la base de sus propios juicios (p. 56). Pese a esta falta de uniformidad terminológica, lo importante es que todos hablan de una relación normativa peculiar entre EPs y CEPs diferente de la que se da entre creencias.

30 Traducimos "entitlement" como "legitimación". Este último término será el que usemos en lo que sigue dado que se presta a menos equívocos que la expresión "justificación inmediata". No obstante, conviene dejar constancia de que la equivalencia entre legitimación y justificación inmediata no es del todo completa. Para Pryor (2007, p. 183) puede haber justificación inmediata de una CEP falible y revocable; mientras que para Burge (2003), aunque las propias representaciones perceptuales son falibles ( p. 526 y ss.), que se dé una legitimación es garantía de cierto éxito (con cabida de margen para un error parcial) en la corrección [correctness] de la CEP legitimada y de la EP que legitima (pp. 530531), éxito que queda garantizado porque una EP contribuye a la legitimación en tanto que sea fiablemente verídica en el entorno normal del sistema perceptual correspondiente (pp. 532 y ss.). 
to haya de considerar sus EPs como sus razones para creer -requisito éste que el conceptualista consideraba imprescindible para que las EPs jueguen un papel normativo-. Burge y Pryor ponen de manifiesto esta idea al afirmar que cuando las CEPs de un sujeto se legitiman a partir de sus EPs se cumplen determinadas normas o reglas epistémicas de las que ese sujeto no tiene que ser consciente (Burge, 2003, pp. 512 y ss; 521; 547) ni ha de seguir de forma deliberativa (Pryor 2005, pp. 195 y ss.). Por su parte, Peacocke (2004, p. 7) afirma que un sujeto «puede estar legitimado a emitir un juicio sin tener la capacidad de pensar acerca de los estados que lo legitiman para emitir ese juicio». Todo esto también forma parte de nuestro enfoque cuando hablamos de sustentación en vez de legitimación. No obstante, pese a la aparente similitud, lo que planteamos no coincide plenamente con lo que plantean estos autores. Para empezar, recordemos que nosotros consideramos el carácter no-conceptual, y por ello no proposicional, de las EPs basándonos en resultados de la psicología de la percepción, como los que proporcionan los experimentos de tipo Sperling en relación a las RMIs. Este es un aspecto que no está en sus propuestas.

Con respecto a los planteamientos de Burge y Pryor se puede establecer una nueva diferencia, epistemológicamente más sustancial. Estos defienden que cuando una EP ${ }^{31}$ legitima una determinada CEP está proporcionando una garantía inmediata de esa CEP. La inmediatez de la garantía es pues un requisito para hablar de legitimación. 32 Para ellos la legitimación no requiere de creencias, y se da en ausencia de razones en contra (Burge, 2003, pp. 526 y 546). En cambio, lo que nosotros entendemos por 'sustentar epistémicamente' no implica una garantía inmediata. Ha de tenerse en cuenta que para nosotros el sustento no queda desvinculado de una relación inferencial, la que se da entre las HPDs y las HPNDs, dado el bagaje de creencias, para concluir una CEP. Así pues, la garantía que proporciona la sustentación epistémica no es inmediata dado que el bagaje de fondo del sujeto siempre está involucrado en la mencionada relación inferencial. Es cierto que Burge y Pryor hacen jugar un papel al bagaje de creencias en la legitimación, pero es meramente el de anular tal legitimación cuando hay razones del bagaje en contra.

Finalmente, no queda claro si conforme a las propuestas de Burge y Pryor las EPs ilusorias pueden proporcionar legitimación. En la nuestra sí pueden sustentar. Recuérdese lo dicho acerca de la ilusión de Müller-Lyer. Téngase en cuenta que dada esa experiencia ilusoria, incluso si la creencia fijada fuera incorrecta (p.e., "las lineas son de diferente longitud"), habría sustentación de esa creencia a la luz del bagaje de creencias disponibles del sujeto (seguramente por falta de creencias que permitan descartar la HPD que desencadene la experiencia ilusoria).

\footnotetext{
31 A partir de aquí damos por supuesto que puede tratarse de una secuencia de EPs.

32 El requisito de inmediatez es explícito en Pryor (2005), y todo apunta a que es la manera en que Burge (2003) entiende la legitimación.
} 
La posición de Peacocke (2004) podría parecer más próxima a la nuestra, ya que él habla de legitimación proporcionada por ilusiones, o en general por EPs cuyos contenidos no son correctos. Pero también con su propuesta hay diferencias significativas. Peacocke (2004, pp. 101-102) distingue tres variedades de legitimación perceptual:

(i) básica, que se daría mediante lo que denomina "contenidos representacionales individuados-por-instancia" [instance-individuated representational contents] de EPs;

(ii) secuencial corroborativa, que se da mediante una secuencia de EPs que sea coherente, incluidas secuencias de EPs con contenidos individuados-porinstancia, y que sirve para incrementar el grado de confirmación de una CEP;

(iii) informacional, que está proporcionada por EPs cuyo contenido no es individuado-por-instancia y está influido por el bagaje de información previo del sujeto (recuerdos, conocimiento, testimonios, etc.).

La legitimación básica sería la legitimación directa de una CEP que dé cuenta del correspondiente contenido perceptual individuado-por-instancia. En la medida en que tal legitimación directa se deba entender como inmediata sería diferente a nuestra noción de 'sustentación', por las razones ya expuestas en relación con las propuestas de Pryor y de Burge. Toda otra legitimación sería derivada, ya sea por la vía secuencial ya por la vía informacional (o por una combinación de ambas), incluida la legitimación que se pueda producir en el caso de ilusiones (como la de Müller-Lyer). Respecto a la legitimación informacional conviene señalar que, aunque nosotros defendemos que el contenido de una EP de un sujeto puede verse afectada por EPs previas de dicho sujeto (dado un aprendizaje perceptual), sin embargo no compartimos la idea (generalizada) de que otras creencias asumidas por ese sujeto pueden afectar al contenido de una EP. Por el contrario, entendemos (como Fodor) que las EPs y sus contenidos no están afectados por el bagaje de creencias (ver arriba III.1).

Ante todo, debemos decir que la noción de contenido individuado-por-instancia que maneja Peacocke (2004) no es demasiado clara. Sin embargo, el carácter central que tiene en su propuesta requeriría que la delimitara con más precisión. Lo más que Peacocke proporciona al respecto es, por un lado, la indicación de que ciertos contenidos de experiencia perceptiva espaciales (concerniendo a asuntos como distancia, dirección, forma y tamaño) y otros temporales serían de esa índole, y tal vez los relativos a texturas de superficies, colores e iluminación, y un rango (totalmente indeterminado) de propiedades materiales; $y$, por otro lado, proporciona como ejemplos de contenidos que no serían (directamente) individuados-por-instancia los 
de ver un mueble como siendo sueco, o ver un objeto como un ordenador Mac, o ver que alguien está triste o encantado. Llamemos a los primeros, teniendo presente un comentario de Peacocke (2004, p. 68), contenidos de EPs constitutivamente básicos. ${ }^{33}$ Pero con independencia de la escasa delimitación de lo que constituirían tales contenidos, el mayor problema es que la idea de contenidos constitutivamente básicos tal y como la presenta Peacocke resulta escasamente satisfactoria a nuestro entender. De las consideraciones de Peacocke respecto de dichos contenidos cabe concluir que o bien (a) en los procesos perceptivos de un sujeto se dan frecuentemente EPs cuyos únicos contenidos son constitutivamente básicos ${ }^{34}$, o bien (b) que dada una EP quepa desagregar su contenido total para obtener contenidos parciales entre los que estarían los constitutivamente básicos como contenidos de esa EP. Pero de esta disyuntiva entendemos que (a) resulta implausible. Si como hemos defendido, las RMIs proporcionan las unidades discretas de la experiencia perceptual, nada indica que el único contenido de una RMI tenga que ser constitutivamente básico en el sentido de Peacocke. Respecto a (b) tampoco resulta más aceptable. El problema estriba en considerar que el contenido global per se de una EP sea desagregable en contenidos parciales de esa EP. Por el contrario, entendemos que, dada una EP -por ejemplo, una RMI en tanto que unidad discreta de la experiencia perceptual-, no hay diversos contenidos (no-conceptuales) de esa EP desagregados. Otra cosa es que al único contenido global de una EP de un sujeto se le haga corresponder varios contenidos proposicionales (y, por tanto, conceptuales) de CEPs con los que dar cuenta de aspectos del contenido de esa EP. Son los contenidos conceptuales de CEPs los que nos permiten establecer disecciones en relación con el contenido no-conceptual de la EP correspondiente, pero el contenido per se de esa EP no se da desagregado. 35

\footnotetext{
33 Los contenidos constitutivamente básicos sirven a Peacocke (2004, p. 70) para establecer una distinción entre conceptos observacionales (p.e, conceptos espaciales como 'estar recto', temporales, texturas (de superficies), colores, ...) y los no- observacionales (como 'ordenador Mac', 'mueble sueco', '(sujeto) triste', 'soldado', 'juez', ...), en el sentido de que los no-observacionales tienen compromisos que van más allá de lo que está involucrado en EPs individuadas-por-instancia..

34 Que en el flujo de experiencia de un sujeto se diera ocasionalmente alguna EP cuyo único contenido fuera constitutivamente básico no sería significativo para el papel epistémico asegurador de la corrección masiva de nuestras percepciones que Peacocke quiere otorgar a ese tipo de EPs.

35 Ésta última parece ser la manera de presentar las cosas por parte de Burge (2003), y en ese aspecto nos sentimos más próximos a su planteamiento. De hecho, Burge (2003, IX) establece una distinción entre conceptos observacionales (como conceptos para formas, posiciones, movimientos, texturas (de superficies), colores, ...) y no-observacionales (como 'tomate', 'amplificador', 'mercurio', 'cáncer', 'coche', 'presidente') que es similar a la de Peacocke, en el sentido de que un concepto observacional está directamente asociado a aspectos de la representación perceptual que están individuados directamente por componentes externos (objetos particulares, propiedades-token, relaciones-token, eventostoken y situaciones particulares). Pero Burge no postula para ello contenidos constitutivamente básicos de EPs, más bien defiende que el contenido de una EP puede dar lugar a conceptualizaciones y
} 
Más allá de sus similitudes y diferencias respecto al resto de posturas, nuestra propuesta, dada la forma en que presenta el rol epistémico de la experiencia perceptual, se sitúa en concordancia con la idea, que se remonta al propio Evans (1982, p. 158), de que las EPS no-conceptuales sólo cumplen un papel epistémico normativo por estar a disposición de un sujeto con capacidades conceptuales. ${ }^{36}$ Este es un punto de vista que, no obstante, McDowell (1994a, pp. 54-55) interpreta como una especie de subterfugio con el que Evans trataría de evitar la objeción de recaída en el mismo mito de lo dado en el que había caído el fundamentalismo clásico. Por ello, y para terminar, hemos de preguntarnos si nuestra propuesta, siendo de corte no-conceptualista, está expuesta a una objeción semejante. Creemos que la respuesta ha de ser negativa si tenemos presente una vez más que para nosotros sustentar epistémicamente no es justificar en base a EPs consideradas como razones genuinas o razones reconocidas por el sujeto como tales. No obstante sustentar sí va vinculado inexorablemente a una relación inferencial en la que está involucrada el bagaje de creencias del sujeto. Dado el papel de tal bagaje, debe resultar claro que no se trata de un proceso de una sola dirección, por lo que no estaríamos ante una justificación en la línea del fundamentismo clásico. Ni siquiera se trata de un fundamentismo menos estricto - como el que parece corresponde a las propuestas de Burge (2003), Peacocke (2004) y Pryor (2005)-. Pero no por ello se puede concluir que estemos sin más ante una especie de coherentismo. Quizás nuestro planteamiento constituya una vía intermedia que, haciendo uso de la expresión acuñada por Haack (1994), podríamos calificar de fundaherentista.

\section{Referencias bibliográficas}

BaloG, K. (2009): “Jerry Fodor on Non-conceptual Content”, Synthese, 170, pp. 311-320.

Bermúdez, J.L. (1995): "Nonconceptual Content: From Perceptual Experience to Subpersonal Computational States", Mind and Language, 10 (4), pp. 333-369. (vers. util. en Gunther, Y. H. (eds.) (2003): Essays on nonconceptual content, Cambridge (Mass.), The MIT Press, pp. 183-216).

Bermúdez, J.L. (1998): The Paradox of Self-Consciousness, Cambridge (Mass.), MIT Press.

creencias más básicas (en las que sólo intervengan conceptos observacionales) y otras menos básicas (en las que intervengan conceptos no-observacionales).

36 Burge (2003, p. 530) comparte esta idea al afirmar que la garantía epistémica que generan las EPs no conceptuales sólo emerge al formar parte éstas de un sistema psicológico que incluya creencias y actos mentales. Hay que apuntar que Burge (2003, p. 519) defiende que el ámbito de posesión de creencias no se restringe al ser humano, sino que abarca primates y otros mamíferos superiores. 
Bermúdez, J.L. y Cahen, A. (2003/2010): "Nonconceptual Mental Content", The Stanford Encyclopedia of Philosophy (Spring 2010 Edition), en Zalta, E. N. (ed.), URL=http://plato.stanford.edu/archives/spr2010/entries/content-nonconceptual.

Becker, M.W., Pashler, H. y Anstis, S. M. (2000): “The Role of Iconic Memory in Change-detection Tasks", Perception, 29, pp. 273-286.

Brewer, B. (1999): Perception and Reason, Oxford, Oxford U.P.

Brewer, B. (2005): “Does Perceptual Experience Has Conceptual Content?”, en

Steup, M. y Sosa, E. (eds.), Contemporary Debates in Epistemology, Malden (Mass.)/Oxford/Carlton (Victoria), Blackwell, pp. 217-230.

Burge, T. (2003): "Perceptual Entitlement", en Philosophy and Phenomenological Research 67 (3): pp. 503-548.

Coltheart, M. (1983): "Ecological Necessity of Iconic Memory", Behavioral and Brain Sciences, 6, pp. 17-18.

Crespo, A. (2006): Cognición Humana. Mente, Ordenadores y Neuronas, Madrid, Editorial Centro de Estudios Ramón Areces.

DAVIDSON, D. (1983): "A Coherence Theory of Truth and Knowledge", en Henrich,

D. (ed.), Kant oder Hegel?, Stuttgart: Klett-Cotta (vers. util. en Sosa, E.; Kim, S.; Fantl, J. y McGrath, M. (eds.) (2000): Epistemology. An Antology, Malden (Mass.)/Oxford, Blackwell, pp. 124-133).

Evans, G. (1982): The Varieties of Reference, Oxford, Clarendon Press.

FernÁndez-Duque, D. y Thornton, I. (2000): "Change Detection Without Awareness: Do Explicit Reports Underestimate the Representation of Change in Visual System?", Visual Cognition, 7, pp. 324-344.

Fodor, J. A. (1983): The Modularity of Mind, Cambridge (Mass.)/London, MIT Press.

Fodor, J. A. (1984): “Observation Reconsidered”, Philosophy of Science, 51, pp. 23-43.

Fodor, J. A. (2007): “The Revenge of the Given", en McLaughlin, B.P. y Cohen, J. (eds.) Contemporary Debates in Philosophy of Mind, Malden (Mass.)/Oxford/Carlton (Victoria), Blackwell, pp. 105-116.

Fodor, J. A. (2008): LOT2.The Language of Thought Revisited, Oxford, Oxford U.P.

Gunther, Y. H. (ed.) (2003): Essays on Nonconceptual Content, Cambridge (Mass.), MIT Press.

HaACK, S. (1994): Evidence and Enquiry: Towards Reconstruction in Epistemology, Oxford, Blackwell.

Heck, R. (2000): "Nonconceptual Content and the "Space of Reasons", en The Philosophical Review, 109, No 4: pp. 483-523. 
HECK, R. (2007): “Are There Different Kinds of Content?”, en McLaughlin, B.P. y Cohen, J. (eds.) Contemporary Debates in Philosophy of Mind, Malden (Mass.)/Oxford/Carlton (Victoria), Blackwell, pp. 117- 137.

HannA, R. (2005): "Kant and Nonconceptual Content" en European Journal of Philosophy, 13, 247-290

Hayнoe, M., Bensinger, D. y Ballard, D. (1998): "Task Constraints in Visual Working Memory", Vision Research, 38, pp. 125-137.

McDowell, J. (1994a): Mind and World. Cambridge (Mass), Harvard U. P.

McDowell, J. (1994b): "The Content of Perceptual Experience", The Philosophical Quarterly, vol. 44, no 175, pp. 190-205.

McDowell, J. (2009): Having The World In View: Essays on Kant, Hegel, and Sellars, Cambridge (Mass.)/London, Harvard U.P.

Peacocke, C. (1992): A Study of Concepts, Cambridge (Mass.), MIT Press.

Peacocke, C. (2001): “Does Perception Have a Nonconceptual Content?", Journal of Philosophy, 98, pp. 239-264.

Peacocke, C. (2004): The Realm of Reason, Oxford, Oxford University Press.

PRYOR, J. (2005): “There is Immediate Justification" en Steup, M. y Sosa, E. (eds.) Contemporary Debates in Epistemology, Malden, MA: Blackwell, pp. 181-202.

Sellars, W. (1963): Science, Perception and Reality, London, Routledge and Kegan Paul.

Sperling, G. (1960): "The Information Available in Brief Visual Presentations", Psychological Monographs: General and Applied, 74 (11, No. 498), pp. 1-29. Toribio, J. (2009): “Contenido No-conceptual”, en Quesada, D. (ed.) Cuestiones de Teoría del Conocimiento, Madrid, Tecnos, pp. 250-275.

José Luís Falguera

Departamento de Lógica y Filosofía Moral

Universidad de Santiago de Compostela

joseluis.falguera@usc.es

Santiago Peleteiro

Universidad de Santiago de Compostela

pelaosanti@hotmail.com 